Research Article

\title{
Electrostatics in the Surroundings of a Topologically Charged Black Hole in the Brane
}

\author{
Alexis Larrañaga, ${ }^{1}$ Natalia Herrera, ${ }^{2}$ and Sara Ramirez $^{2}$ \\ ${ }^{1}$ National Astronomical Observatory, National University of Colombia, Bogota 11001000, Colombia \\ ${ }^{2}$ Department of Physics, National University of Colombia, Bogota 11001000, Colombia
}

Correspondence should be addressed to Alexis Larrañaga; ealarranaga@unal.edu.co

Received 27 December 2013; Revised 21 January 2014; Accepted 23 January 2014; Published 27 February 2014

Academic Editor: Christian Corda

Copyright ( 2014 Alexis Larrañaga et al. This is an open access article distributed under the Creative Commons Attribution License, which permits unrestricted use, distribution, and reproduction in any medium, provided the original work is properly cited. The publication of this article was funded by SCOAP ${ }^{3}$.

We determine the expression for the electrostatic potential generated by a point charge held stationary in the topologically charged black hole spacetime arising from the Randall-Sundrum II braneworld model. We treat the static electric point charge as a linear perturbation on the black hole background and an expression for the electrostatic multipole solution is given: PACS: 04.70.-s, 04.50.Gh, 11.25.-w, 41.20.-q, 41.90.+e.

\section{Introduction}

The idea of our universe as a brane embedded in a higher dimensional spacetime has recently attracted attention. According to the braneworld scenario, the physical fields (electromagnetic, Yang-Mills, etc.) in our 4-dimensional universe are confined to the three-brane and only gravity propagates in the bulk spacetime. One of the most interesting scenarios is Randall-Sundrum II model in which it is considered a $\mathbb{Z}_{2}$-symmetric, 5-dimensional, asymptotically anti-deSitter bulk [1] and our brane is identified as a domain wall. The 5-dimensional metric can be written in the general form $d s^{2}=e^{-F(y)} \eta_{\mu \nu} d x^{\mu} d x^{\nu}+d y^{2}$ and due to the appearance of the warp factor, it reproduces a large hierarchy between the scale of particle physics and gravity. Moreover, even if the fifth dimension is uncompactified, standard 4-dimensional gravity on the brane is reproduced. However, due to the correction terms coming from the extradimensions, significant deviations from general relativity may occur at high energies [2-4].

As is well known, in general relativity the exterior spacetime of a spherical compact object is described by Schwarzschild solution. In the braneworld scenario, the high energy corrections to the energy density together with Weyl stresses from bulk gravitons imply that the exterior metric of a spherical compact object on the brane is no longer described by Schwarzschild metric. In fact, black hole solutions in the braneworld model are particularly interesting because they have considerably richer physical aspects than black holes in general relativity [5-11]. The first solutions describing static and spherically symmetric exterior vacuum solutions of the braneworld model were proposed by Dadhich et al. [12] and Germani and Maartens [13] and later they were generalised by Chamblin et al. [14] and revisited by Sheykhi and Wang [15]. This kind of solutions carries a topological charge arising from the bulk Weyl tensor and the line element resembles Reissner-Nördstrom solution, with the tidal Weyl parameter playing the role of the electric charge. In order to obtain this solution, there was the null energy condition imposed on the three-brane for a bulk having nonzero Weyl curvature.

On the other hand, the generation of an electromagnetic field by static sources in black hole backgrounds has been considered in several papers beginning with the studies of Copson [16], Cohen and Wald [17], and Hanni and Ruffini [18] where they discussed the electric field of a point charge in Schwarzschild background. Afterwards, Petterson [19] and later Linet [20] studied the magnetic field of a current loop surrounding a Schwarzschild black hole. More recently, similar studies have been performed in other background 
geometries including charged black holes of general relativity and black holes with Brans-Dicke modifications or with conical defects [21-25].

In this paper we are interested in obtaining an expression for the electrostatic potential generated by a point charge held stationary in the region outside the event horizon of a braneworld black hole. There are several vacuum solutions of the spherically symmetric static gravitational field equations on the brane with arbitrary parameters which depend on properties of the bulk or that are simply put in by using general physical considerations. At the present, it is theoretically not known whether these parameters should be universal over all braneworld black holes, or whether each separate black hole may have different values of them. Similarly, there is not a single complete solution in the sense that the metric in the bulk is uniquely known. Since this situation is unsatisfactory from a theoretical point of view, it may be useful to investigate more closely the observational effects of the black hole properties. Specifically, we consider the effects due to the projections of the Weyl tensor and how they specify the corrections on the electrostatic potential. Since the generic form of the Weyl tensor in the full 5-dimensional spacetime is yet unknown, the effects of known solutions must be studied on a case-by-case basis. Although one can, in principle, constrain the projections, this only yields very mild constraints on the 5-dimensional Weyl tensor [26, 27]. Therefore we decided to derive the electrostatic potential generated by a point charge held stationary in the outside region of the event horizon of the particular solution in the Randall-Sundrum braneworld model obtained by Dadhich et al. [12] and revisited by Sheykhi and Wang [15]. In this case, tidal charge is arising via gravitational effects from the fifth dimension; that is, it is arising from the projection onto the brane of free gravitational field effects in the bulk, and is this term the one that will modify the electrostatic potential produced by the particle. We obtain the corrections, due to the topological charge arising from the bulk Weyl tensor, to the electrostatic potential and deduce the necessary correction to incorporate Gauss's law. Finally we also present the solution of Maxwell equations in the form of series of multipoles.

\section{The Topologically Charged Black Hole in the Braneworld}

The gravitational field on the brane is described by the Gauss and Codazzi equations of 5-dimensional gravity [2],

$$
G_{\mu \nu}=-\Lambda g_{\mu \nu}+8 \pi G T_{\mu \nu}+\kappa_{5}^{4} \Pi_{\mu \nu}-E_{\mu \nu}
$$

where $G_{\mu \nu}=R_{\mu \nu}-(1 / 2) g_{\mu \nu} R$ is the 4-dimensional Einstein tensor and $\kappa_{5}$ is the 5 -dimensional gravity coupling constant,

$$
\kappa_{5}^{4}=\left(8 \pi G_{5}\right)^{2}=\frac{48 \pi G}{\tau},
$$

with $G_{5}$ the gravitational constant in five dimensions and $\Lambda$ is the 4-dimensional cosmological constant that is given in terms of the 5-dimensional cosmological constant $\Lambda_{5}$ and the brane tension $\tau$ by

$$
\Lambda=\frac{\kappa_{5}^{2}}{2}\left(\Lambda_{5}+\frac{\kappa_{5}^{2}}{6} \tau^{2}\right) .
$$

$T_{\mu \nu}$ is the stress-energy tensor of matter confined on the brane, $\Pi_{\mu \nu}$ is a quadratic tensor in the stress-energy tensor given by

$$
\begin{aligned}
& \Pi_{\mu \nu} \\
& \quad=\frac{1}{12} T T_{\mu \nu}-\frac{1}{4} T_{\mu \sigma} T_{\nu}^{\sigma}+\frac{1}{8} g_{\mu \nu}\left(T_{\alpha \beta} T^{\alpha \beta}-\frac{1}{3} T^{2}\right)
\end{aligned}
$$

with $T=T_{\sigma}^{\sigma}$, and $E_{\mu \nu}$ is the projection of the 5-dimensional bulk Weyl tensor $C_{A B C D}$ on the brane $\left(E_{\mu \nu}=\delta_{\mu}^{A} \delta_{\nu}^{B} C_{A B C D}\right.$ $n^{A} n^{B}$ with $n^{A}$ the unit normal to the brane). $E_{\mu \nu}$ encompasses the nonlocal bulk effect and it is traceless, $E_{\sigma}^{\sigma}=0$.

Considering the Randall-Sundrum scenario with

$$
\Lambda_{5}=-\frac{\kappa_{5}^{2}}{6} \tau^{2}
$$

which implies

$$
\Lambda=0
$$

the four-dimensional Gauss and Codazzi equations for an arbitrary static spherically symmetric object have been completely solved on the brane, obtaining a black hole type solution of the field equations (1) with $T_{\mu \nu}=0$, given by the line element $[12,14,15,28]$

$$
d s^{2}=h(r) d t^{2}-\frac{d r^{2}}{h(r)}-r^{2} d \Omega^{2},
$$

where $d \Omega^{2}=d \theta^{2}+\sin ^{2} \theta d \varphi^{2}$ and

$$
h(r)=1-\frac{2 G M}{r}+\frac{\beta}{r^{2}} .
$$

For this model, the expression of the projected Weyl tensor transmitting the tidal charge stresses from the bulk to the brane is

$$
E_{t}^{t}=E_{r}^{r}=-E_{\theta}^{\theta}=-E_{\varphi}^{\varphi}=\frac{\beta}{r^{4}} .
$$

This result shows that the parameter $\beta$ can be interpreted as a tidal charge associated with the bulk Weyl tensor and therefore, there is no restriction on it to take positive as well as negative values (other interpretations consider $\beta$ as a five-dimensional mass parameter as discussed in [14]). The induced metric in the domain wall presents horizons at the radii (taking $G=1$ ). Consider the following:

$$
r_{ \pm}=M \pm \sqrt{M^{2}-\beta} .
$$


For $\beta \geq 0$ there is a direct analogy to the ReissnerNördstrom solution, showing two horizons that, as in general relativity, both lie inside the Schwarzschild radius $2 M$; that is,

$$
0 \leq r_{-} \leq r_{+} \leq r_{s}
$$

Clearly, there is an upper limit on $\beta$, namely,

$$
0 \leq \beta \leq M^{2} \text {. }
$$

However, there is nothing to stop us choosing $\beta$ to be negative. This intriguing new possibility is impossible in Reissner-Nördstrom case and leads to only one horizon, $r_{*}$, lying outside the corresponding Schwarzschild radius,

$$
r_{*}=M+\sqrt{M^{2}+|\beta|}>2 M
$$

In this case, the single horizon has a greater area that its Schwarzschild counterpart. Thus, one concludes that the effect of the bulk producing a negative $\beta$ is to strengthen the gravitational field outside the black hole (obviously it also increases the entropy and decreases the Hawking temperature but these facts are not important in this paper).

\section{The Electrostatic Field of a Point Particle}

Copson [16] and Linet [20] found the electrostatic potential in a closed form of a point charge at rest outside the horizon of a Schwarzschild black hole and that the multipole expansion of this potential coincides with the one given by Cohen and Wald [17] and by Hanni and Ruffini [18]. In this section we will investigate this problem in the background of the topologically charged black hole (7).

According to the braneworld scenario, we will consider that the physical fields are confined to the three brane and if the electromagnetic field of the point particle is assumed to be sufficiently weak so its gravitational effect is negligible, and the Einstein-Maxwell equations reduce to Maxwell's equations confined in the curved background of the brane (7). These are written as

$$
\nabla_{\rho} F^{\rho \mu}=4 \pi J^{\mu}
$$

where

$$
F_{\mu \nu}=\partial_{\mu} A_{\nu}-\partial_{\nu} A_{\mu}
$$

with $A^{\mu}$ the electromagnetic vector potential and $J^{\mu}$ the current density. Considering that the test charge $q$ is held stationary at the point $\left(r_{0}, \theta_{0}, \varphi_{0}\right)$, the associated current density $J^{i}$ vanishes while the charge density $J^{0}$ is given by

$$
J^{0}=\frac{q}{r_{0}^{2} \sin \theta} \delta\left(r-r_{0}\right) \delta\left(\theta-\theta_{0}\right) \delta\left(\varphi-\varphi_{0}\right) .
$$

From now on, we will assume that the charge $q$ is held outside the black hole; that is, $r_{0}>r_{+}$in the case $\beta \geq 0$ and $r_{0}>r_{*}$ in the case $\beta<0$. We will not consider here the possibility of having the charge inside the event horizon because; as well known, this construction leads to the description of a charged black hole of the ReissnerNördstrom type [15].

The spatial components of the potential vanish, $A^{i}=0$, while the temporal component $A^{0}=\phi$ is determined by the $\mu=0$ component of (14), giving the differential equation:

$$
\begin{aligned}
\frac{\partial}{\partial r}\left(r^{2} \frac{\partial \phi}{\partial r}\right) & \\
& +\left(1-\frac{2 M}{r}+\frac{\beta}{r^{2}}\right)^{-1} \\
& \times\left[\frac{1}{\sin \theta} \frac{\partial}{\partial \theta}\left(\sin \theta \frac{\partial \phi}{\partial \theta}\right)+\frac{1}{\sin ^{2} \theta} \frac{\partial^{2} \phi}{\partial \varphi^{2}}\right] \\
= & -r^{2} 4 \pi J^{0} .
\end{aligned}
$$

In order to solve this equation we will perform the substitutions

$$
\begin{gathered}
r=\widetilde{r}+r_{-}, \\
\phi(r, \theta, \varphi)=\frac{r-r_{-}}{r} \widetilde{\phi}\left(r-r_{-}, \theta, \varphi\right)
\end{gathered}
$$

which turn the differential equation into

$$
\begin{aligned}
& \frac{\partial}{\partial \tilde{r}}\left(\tilde{r}^{2} \frac{\partial \tilde{\phi}}{\partial \widetilde{r}}\right) \\
& \quad+\left(1-\frac{2 m}{\tilde{r}}\right)^{-1}\left[\frac{1}{\sin \theta} \frac{\partial}{\partial \theta}\left(\sin \theta \frac{\partial \tilde{\phi}}{\partial \theta}\right)+\frac{1}{\sin ^{2} \theta} \frac{\partial^{2} \tilde{\phi}}{\partial \varphi^{2}}\right] \\
& =-\frac{4 \pi \tilde{q}}{\sin \theta} \delta\left(\tilde{r}-\tilde{r}_{0}\right) \delta\left(\theta-\theta_{0}\right) \delta\left(\varphi-\varphi_{0}\right),
\end{aligned}
$$

where $m=\sqrt{M^{2}-\beta}$ and $\tilde{q}=q \widetilde{r}_{0} /\left(\widetilde{r}_{0}+r_{-}\right)$. Note that, in the extremal case $m=0$, or equivalently $M^{2}=\beta$, (19) becomes Laplace's equation in Minkowski spacetime. On the other hand, when $m \neq 0$ (including values with $0 \leq \beta<M^{2}$ as well as $\beta<0)$, (19) is formally identical to the partial differential equation for the electrostatic potential in the Schwarzschild spacetime. Hence, assuming $\theta_{0}=0, \varphi_{0}=0$ and proceeding in analogy with Copson [16], we find that the solution of (17), after using the substitutions (18), is

$$
\phi_{C}(r, \theta)=\frac{q}{r_{0} r} \frac{(r-M)\left(r_{0}-M\right)-\left(M^{2}-\beta\right) \cos \theta}{\sqrt{(r-M)^{2}+\left(r_{0}-M\right)^{2}-2(r-M)\left(r_{0}-M\right) \cos \theta-\left(M^{2}-\beta\right) \sin ^{2} \theta}} .
$$


This solution describes, as stated before, the potential for a charge $q$ situated at the point $\left(r_{0}, 0,0\right)$ and it is regular for any value of $r$ outside the event horizon, except at the position of the point charge. However, as shown by Linet [20] and Léauté and Linet [21], it is easy to see that this solution includes another source. Note that, for $r \rightarrow \infty$, the potential $\phi_{C}$ takes the asymptotic form

$$
\phi_{C}(r, \theta) \longrightarrow \frac{q}{r}\left(1-\frac{M}{r_{0}}\right)
$$

and consequently, by virtue of Gauss's theorem, there is a second charge with value $-q M / r_{0}$. Solution (20) has only the source $q$ outside the horizon and thus the second charge must lie inside the horizon. Moreover, since the only electric field which is regular outside the horizon is spherical symmetric [29], the electrostatic potential for our physical system will be of the form

$$
\phi(r, \theta)=\phi_{C}(r, \theta)+\frac{q}{r} \frac{M}{r_{0}} .
$$

The electrostatic solution can be analysed for all values $r$. Considering this equation, it is clear that when the charge $q$ is approaching the outer horizon $r_{+}$in the case $\beta \geq 0$ or the horizon $r_{*}$ in the case $\beta<0$, the electrostatic potential $\phi$ tends to become spherically symmetric, recovering a charged black hole of the Reissner-Nördstrom type as stated before.

When $r_{0}>2 M$, there is only one charge $q$ in the case $\beta \geq 0$ as well as in the case $\beta<0$. However, when $r_{+} \leq r_{0}<$ $2 M$ in the case $\beta \geq 0$, there are also two other charges $q_{1}=$ $q\left(1-2 M / r_{0}\right)$ and $q_{2}=-q_{1}$ located at $\left(r=2 M-r_{0}, \theta=\pi\right)$ and $r=0$, respectively. This behaviour is obviously not present for $\beta<0$ because $r_{*}>2 M$ as shown in Section 2 of this paper.

Finally, in order to write formally the electrostatic potential of a charge $q$ located at the arbitrary point with coordinates $\left(r_{0}, \theta_{0}, \varphi_{0}\right)$, we simply replace the term $\cos \theta$ by the function $\lambda(\theta, \varphi)=\cos \theta \cos \theta_{0}+\sin \theta \sin \theta_{0} \cos \left(\varphi-\varphi_{0}\right)$. This gives the general solution

$$
\phi(r, \theta, \varphi)=\frac{q}{r_{0} r} \frac{(r-M)\left(r_{0}-M\right)-\left(M^{2}-\beta\right) \lambda(\theta, \varphi)}{\sqrt{(r-M)^{2}+\left(r_{0}-M\right)^{2}-2(r-M)\left(r_{0}-M\right) \lambda(\theta, \varphi)-\left(M^{2}-\beta\right)\left[1-\lambda^{2}(\theta, \varphi)\right]}}+\frac{q}{r} \frac{M}{r_{0}} .
$$

3.1. Multipole Expansion. If the angular part of the potential in (19) is expanded in terms of Legendre polynomials in $\cos \theta$, one may obtain the well-known electric multipole solutions. Using again the substitutions (18), the radial parts of the two linearly independent multipole solutions of (17) are

$$
\begin{aligned}
& g_{l}(r)= \begin{cases}1 & \text { for } l=0 \\
\frac{2^{l} l !(l-1) !\left(M^{2}-\beta\right)^{l / 2}}{(2 l) !} \frac{r^{2}-2 M r+\beta}{r} \frac{d P_{l}}{d r}\left(\frac{r-M}{\sqrt{M^{2}-\beta}}\right) & \text { for } l=1,2, \ldots,\end{cases} \\
& f_{l}(r)=-\frac{(2 l+1) !}{2^{l}(l+1) ! ! !\left(M^{2}-\beta\right)^{(l+1) / 2}} \frac{r^{2}-2 M r+\beta}{r} \frac{d Q_{l}}{d r}\left(\frac{r-M}{\sqrt{M^{2}-\beta}}\right) \text { for } l=0,1,2, \ldots,
\end{aligned}
$$

where $P_{l}$ and $Q_{l}$ are the two types of Legendre functions. These functions satisfy the following:

(1) for $l=0, g_{0}(r)=1$ and $f_{0}(r)=1 / r$;

(2) for all values of $l$, as $r \rightarrow \infty$, the leading term of $g_{l}(r)$ is $r^{l}$ while the leading term of $f_{l}(r)$ is $r^{-(l+1)}$;

(3) as $r \rightarrow r_{+}, g_{l}(r) \rightarrow 0($ for $l \neq 0)$ while $f_{l}(r) \rightarrow$ finite constant. However, $d f_{l} / d r$ blows up for $l \neq 0$ when $r \rightarrow r_{+}$.

The above properties let us infer that only the set of solutions (25) has the correct behaviour at infinity and only the multipole term $l=0$ does not produce a divergent field at the horizon $r=r_{+}$. This set of solutions reproduces Israel's result [29] when $\beta=0$ (i.e., for the Schwarzschild black hole).
As is well known, the gravitational field modifies the electrostatic interaction of a charged particle in such a way that the particle experiences a finite self-force [22, 23, 30-34] whose origin comes from the spacetime curvature associated with the gravitational field. However, even in the absence of curvature, it was shown that a charged point particle $[35,36]$ or a linear charge distribution [37] placed at rest may become subject to a finite repulsive electrostatic self-force (see also [24]). In these references, the origin of the force is the distortion in the particle field caused by the lack of global flatness of the spacetime of a cosmic string. Therefore, one may conclude that the modifications of the electrostatic potential come from two contributions: one of geometric origin and the other of a topological one. In a forthcoming paper we will show that, considering the topologically charged black hole 
described by the line element (7) as the background metric, both kinds of contributions appear in the electrostatic selfforce of a charged particle.

\section{Conflict of Interests}

The authors declare that there is no conflict of interests regarding the publication of this paper.

\section{Acknowledgment}

This work was supported by the Universidad Nacional de Colombia, Hermes Project Code 18140.

\section{References}

[1] L. Randall and R. Sundrum, "An alternative to compactification," Physical Review Letters, vol. 83, no. 23, pp. 4690-4693, 1999.

[2] T. Shiromizu, K.-I. Maeda, and M. Sasaki, "The Einstein equations on the 3-brane world," Physical Review D, vol. 62, no. 2, Article ID 024012, 6 pages, 2000.

[3] M. Sasaki, T. Shiromizuand, and K. Maeda, "Gravity, stability, and energy conservation on the Randall-Sundrum brane world," Physical Review D, vol. 62, Article ID 024008, 8 pages, 2000.

[4] S. Nojiri, S. D. Odintsov, and S. Ogushi, "FriedmannRobertson-Walker brane cosmological equations from the fivedimensional bulk (A)dS black hole," International Journal of Modern Physics A, vol. 17, no. 32, pp. 4809-4870, 2002.

[5] S. Nojiri, O. Obregon, S. D. Odintsov, and S. Ogushi, "Dilatonic brane-world black holes, gravity localization, and Newton's constant," Physical Review D, vol. 62, no. 6, Article ID 064017, 10 pages, 2000.

[6] P. Kanti and K. Tamvakis, "Quest for localized 4D black holes in brane worlds," Physical Review D, vol. 65, no. 8, Article ID 084010, 2002.

[7] R. Casadio, A. Fabbri, and L. Mazzacurati, "New black holes in the brane world?” Physical Review D, vol. 65, no. 8, Article ID 084040, 2002.

[8] R. Casadio and L. Mazzacurati, "Bulk shape of brane-world black holes," Modern Physics Letters A, vol. 18, no. 9, pp. 651660, 2003.

[9] R. Casadio, "On brane-world black holes and short scale physics," Annals of Physics, vol. 307, no. 2, pp. 195-208, 2003.

[10] A. Chamblin, S. W. Hawking, and H. S. Reall, "Brane-world black holes," Physical Review D, vol. 61, no. 6, Article ID 065007, 6 pages, 2000.

[11] R. Casadio and B. Harms, "Black hole evaporation and large extra dimensions," Physics Letters B, vol. 487, no. 3-4, pp. 209214,2000

[12] N. Dadhich, R. Maartens, P. Papadopoulos, and V. Rezania, "Quark mixings in SU(6) X SU(2)(R) and suppression of V(ub)," Physics Letters B, vol. 487, no. 1-2, pp. 104-109, 2000.

[13] C. Germani and R. Maartens, "Stars in the braneworld," Physical Review D, vol. 64, no. 12, Article ID 124010, 2001.

[14] A. Chamblin, H. S. Reall, H.-A. Shinkai, and T. Shiromizu, "Charged brane-world black holes," Physical Review D, vol. 63, no. 6, Article ID 064015, 2001.
[15] A. Sheykhi and B. Wang, "On topological charged braneworld black holes," Modern Physics Letters A, vol. 24, no. 31, pp. 25312538, 2009.

[16] E. Copson, "On electrostatics in a gravitational field," Proceedings of the Royal Society A, vol. 118, pp. 184-194, 1928.

[17] J. M. Cohen and R. M. Wald, "Point charge in the vicinity of a Schwarzschild black hole," Journal of Mathematical Physics, vol. 12, no. 9, pp. 1845-1849, 1971.

[18] R. S. Hanni and R. Ruffini, "Lines of force of a point charge near a schwarzschild black hole," Physical Review D, vol. 8, no. 10, pp. 3259-3265, 1973.

[19] J. A. Petterson, "Magnetic field of a current loop around a schwarzschild black hole," Physical Review D, vol. 10, no. 10, pp. 3166-3170, 1974.

[20] B. Linet, "Electrostatics and magnetostatics in the Schwarzschild metric," Journal of Physics A, vol. 9, no. 7, pp. 1081-1087, 1976.

[21] B. Léauté and B. Linet, "Electrostatics in a Reissner-Nordström space-time," Physics Letters A, vol. 58, pp. 5-6, 1976.

[22] B. Léauté and B. Linet, "Principle of equivalence and electromagnetism," International Journal of Theoretical Physics, vol. 22, no. 1, pp. 67-72, 1983.

[23] B. Léauté and B. Linet, "Self-interaction of an electric or magnetic dipole in the Schwarzschild space-time," Classical Quantum Gravity, vol. 1, no. 1, p. 5, 1985.

[24] J. Spinelly and V. B. Bezerra, "Electrostatic in ReissnerNordström space-time with a conical defect," Modern Physics Letters A, vol. 15, no. 32, pp. 1961-1966, 2000.

[25] M. Watanabe and A. W. C. Lun, "Electrostatic potential of a point charge in a Brans-Dicke Reissner-Nordström field," Physical Review D, vol. 88, Article ID 045007, 11 pages, 2013.

[26] R. Maartens, "Brane-world gravity," Living Reviews in Relativity, vol. 7, pp. 1-99, 2004.

[27] C. S. J. Pun, Z. Kovács, and T. Harko, "Thin accretion disks onto brane world black holes," Physical Review D, vol. 78, no. 8, Article ID 084015, 2008.

[28] C. G. Böhmer, T. Harko, and F. S. N. Lobo, "Wormhole geometries with conformal motions," Classical and Quantum Gravity, vol. 25, no. 7, Article ID 075016, 2008.

[29] W. Israel, "Event horizons in static electrovac space-times," Communications in Mathematical Physics, vol. 8, no. 3, pp. 245260, 1968.

[30] A. Vilenkin, "Self-interaction of charged particles in the gravitational field," Physical Review D, vol. 20, no. 2, pp. 373-376, 1979.

[31] F. Piazzese and G. Rizzi, "On the electrostatic self-force in a Reissner-Nordström spacetime," Physics Letters A, vol. 119, no. 1, pp. 7-9, 1986.

[32] B. Boisseau, C. Charmousis, and B. Linet, "Electrostatic selfforce in a static weak gravitational field with cylindrical symmetry," Classical and Quantum Gravity, vol. 13, no. 7, pp. 1797-1803, 1996.

[33] A. G. Smith and C. M. Will, "Force on a static charge outside a Schwarzschild black hole," Physical Review D, vol. 22, no. 6, pp. 1276-1284, 1980.

[34] A. I. Zel'nikov and V. P. Frolov, "Influence of gravitation on the self-energy of charged particles," Journal of Experimental and Theoretical Physics, vol. 55, no. 2, p. 191, 1982.

[35] B. Linet, "Force on a charge in the space-time of a cosmic string," Physical Review D, vol. 33, no. 6, pp. 1833-1834, 1986. 
[36] A. G. Smith, "Gravitational effects of an infinite straight cosmic string on classical and quantum fields: self-forces and vacuum Fluctuations," in Proceedings of the Symposium of the Formation and Evolution of Cosmic Strings, G. W. Gibbons, S. W. Hawking, and T. Vaschapati, Eds., p. 263, Cambridge University Press, 1990.

[37] E. R. Bezerra de Mello, V. B. Bezerra, C. Furtado, and F. Moraes, "Self-forces on electric and magnetic linear sources in the spacetime of a cosmic string," Physical Review D, vol. 51, no. 12, pp. 7140-7143, 1995. 

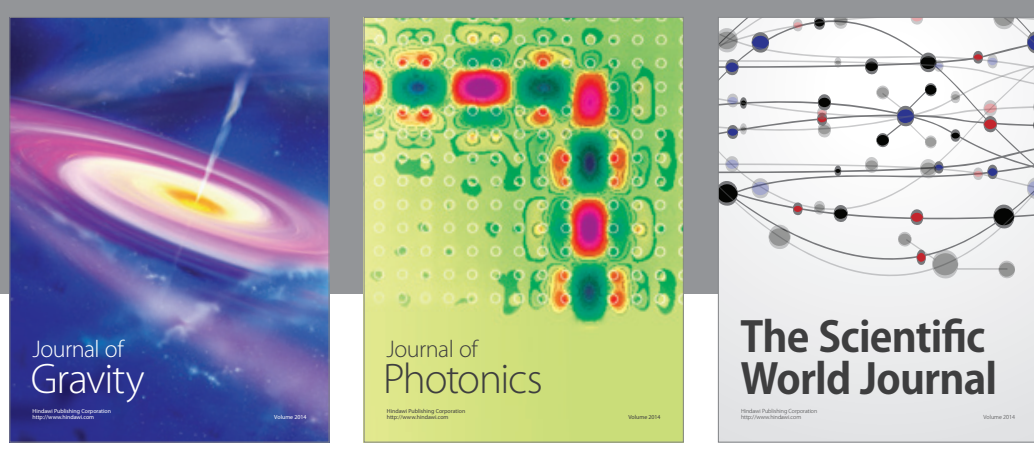

The Scientific World Journal
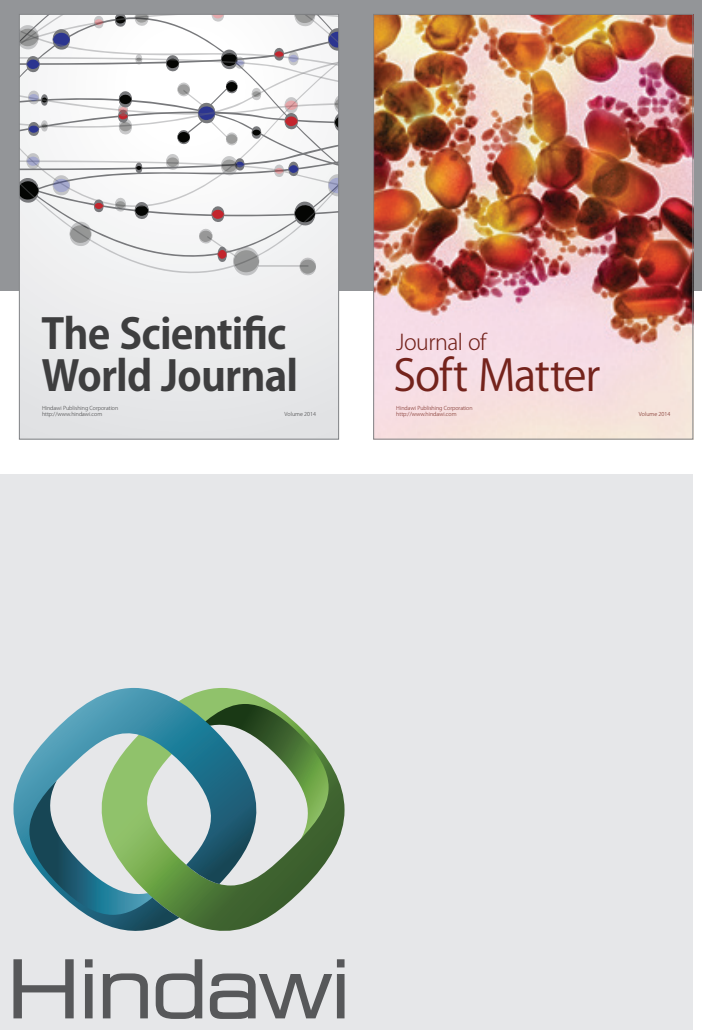

Submit your manuscripts at

http://www.hindawi.com

nternational Journal of

Statistical Mechanics
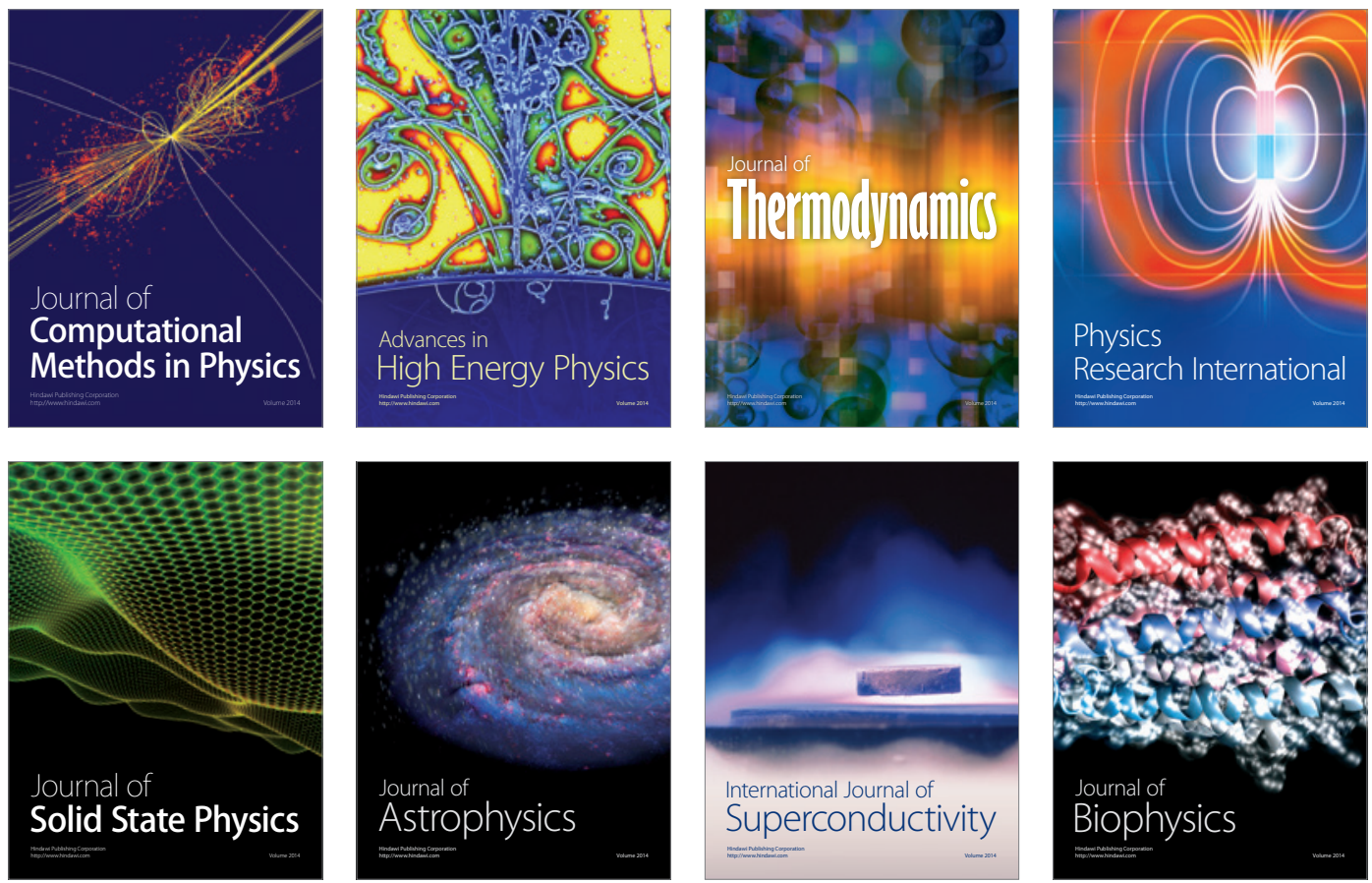
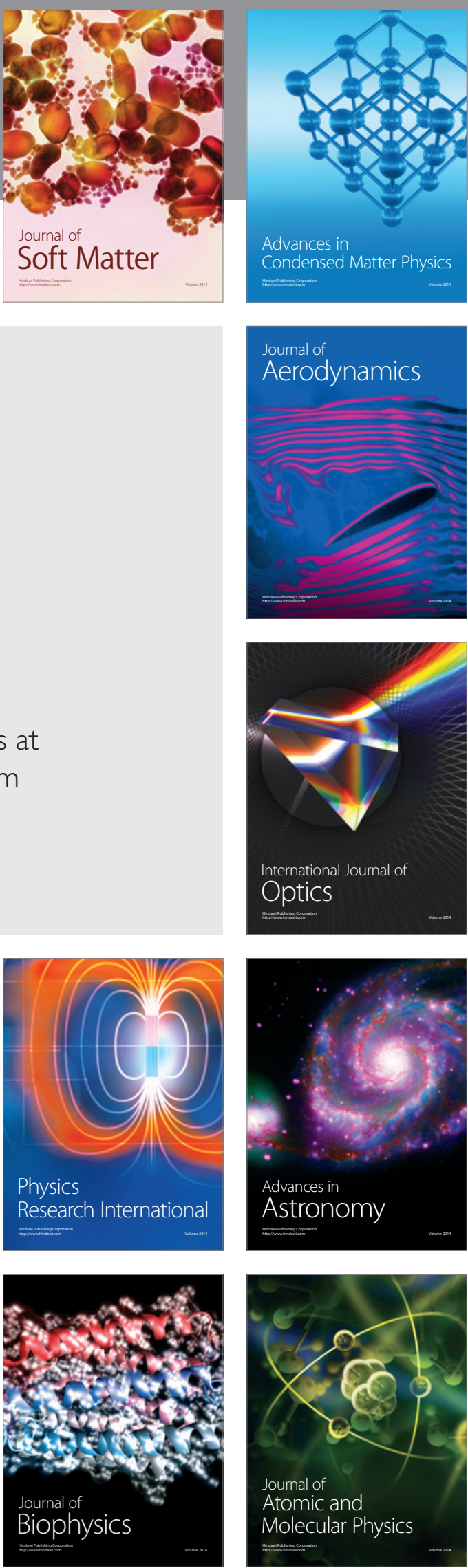